\title{
A Rare Case of Kimura Disease with Bilateral Parotid Involvement
}

\author{
Soo Hyun Woo ${ }^{1}$, Han Koo Kim ${ }^{1}$, Woo Seob Kim, Tae Hui Bae ${ }^{1}$, Mi Kyung Kim² \\ Departments of ${ }^{1}$ Plastic and Reconstructive Surgery and ${ }^{2}$ Pathology, Chung-Ang University Hospital, Chung-Ang University College of \\ Medicine, Seoul, Korea
}

Kimura disease is a rare idiopathic chronic inflammatory disorder. It typically presents in the head and neck area, whereas bilateral involvement is unusual. Its diagnosis requires it to be differentiated from other inflammatory diseases and from head and neck tumors. Treatment methods include conservative management, steroid administration, radiotherapy, and surgery; however, no single treatment of choice has been established. Herein, we report an unusual presentation of Kimura disease with bilateral parotid involvement. This case was treated by surgical excision.

Keywords Head and neck neoplasms / Parotid neoplasms / Angiolymphoid hyperplasia with eosinophilia

\author{
Correspondence: Han Koo Kim \\ Department of Plastic and \\ Reconstructive Surgery, Chung-Ang \\ University Hospital, Chung-Ang \\ University College of Medicine, \\ 102 Heuksuk-ro, Dongjak-gu, Seoul \\ 06973, Korea \\ Tel: +82-2-6299-1628 \\ Fax: +82-2-823-1049 \\ E-mail:hkkiim@cau.ac.kr
}

This article was presented as a poster at the 73rd Congress of the Korean Society of Plastic and Reconstructive Surgeons, November 13-15, 2015 in Seoul, Korea.

No potential conflict of interest relevant to this article was reported.

Received: 10 Nov 2016 • Revised: 27 Feb $2017 \bullet$ Accepted: 21 Mar 2017

pISSN: 2234-6163 • elSSN: 2234-6171 • https://doi.org/10.5999/aps.2017.44.5.439 • Arch Plast Surg 2017;44:439-443

\section{INTRODUCTION}

First described in 1937, Kimura disease (KD) is a rare benign chronic inflammatory swelling of unknown etiology [1]. It usually presents with unilateral swelling in the soft tissue in the head and neck area, including the lymph nodes and salivary glands, and is characterized by eosinophilia and elevated immunoglobulin E (IgE) levels. It typically occurs in Asian men in their second and third decades of life. In most cases of $\mathrm{KD}$, a unilateral soft tissue mass is present, whereas bilateral involvement is rare [2]. $\mathrm{KD}$ is difficult to diagnose, and must be differentiated from inflammatory and neoplastic conditions, tuberculosis, cylindroma, dermatofibrosarcoma protuberans, Kaposi sarcoma, pyogenic granuloma, and other infectious lymph node enlarge- ments [3]. We report a very rare case of $\mathrm{KD}$ with synchronous involvement of the bilateral parotid glands.

\section{CASE}

A 33-year-old Korean man visited our clinic with swelling in both cheeks. He had no notable medical history other than smoking, and his vital signs were stable. The masses in his cheeks had existed for about 10 years and had gradually increased in size. The parotid nodules were intermittently painful, and changes in his facial shape were evident. The masses in the bilateral parotid region were firm and palpable. The patient reported no tenderness or other symptoms, and the overlying skin was normal. He had already undergone fine-needle aspira- 
tion cytology (FNAC) at another hospital before visiting our clinic. KD was suspected because FNAC showed lymphoid hyperplasia and an elevated eosinophil count. A blood test revealed eosinophilia (24\%) and an elevated IgE level ( > 5,000 $\mathrm{kU} / \mathrm{L}$; normal range, $2-100 \mathrm{kU} / \mathrm{L}$ ). Urinalysis results were normal.

Enhanced neck computed tomography (CT) showed soft-tissue mass lesions in both cheeks. The masses were located between the masseter muscles and platysma, adjacent to the parotid gland. They showed mild nonhomogeneous enhancement (Fig. 1) with lymphadenopathy at both level IA and IB. KD was suspected.

The masses were completely excised via transverse incisions in both cheeks, not through a face-lift incision. This was done to ensure easier access and better visibility, in order to avoid facial nerve injury, because the mass was long and horizontally positioned beyond the anterior border of the masseter muscle. Moreover, the patient was not concerned about scar formation. Our preoperative plan was to perform a lumpectomy, including mass excision with partial parotid gland removal where the mass was in contact with the gland, because the masses were adhered to the parotid gland and facial nerve branches. Surgery was performed as planned. We used a facial nerve stimulator to monitor facial nerve injury.

The excised masses measured $3.6 \times 2.4 \times 1.4 \mathrm{~cm}^{3}$ (from the left cheek) and $4.8 \times 3.5 \times 1.5 \mathrm{~cm}^{3}$ (from the right cheek) (Fig. 2). Histopathology confirmed the diagnosis of $\mathrm{KD}$. Histopathologic examination showed lymphoid hyperplasia with massive eosinophil infiltration and dense lymphoeosinophilic infiltration in a fibrotic background (Fig. 3). Postoperative recovery was good,

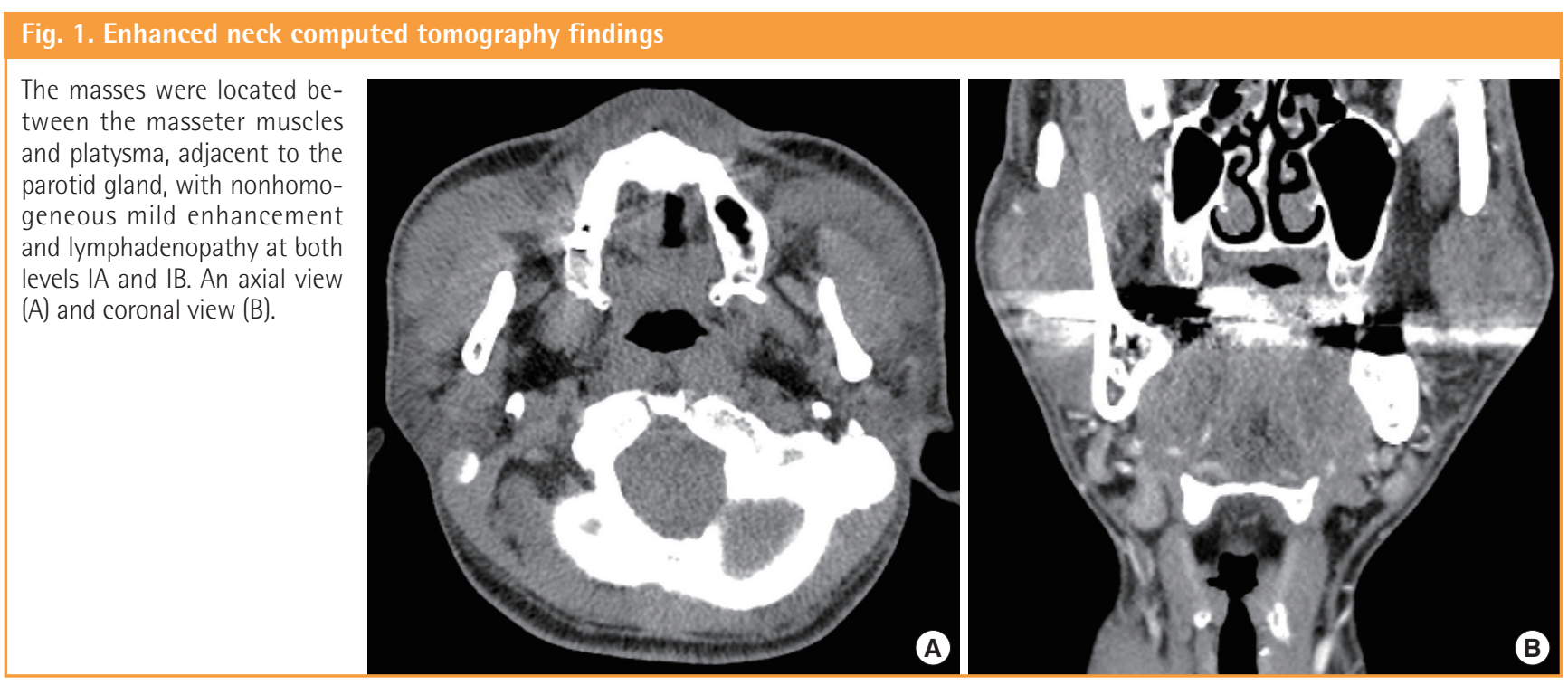

Fig. 2. Intraoperative photographs

Excised masses from the left cheek (A) and the right cheek (B).

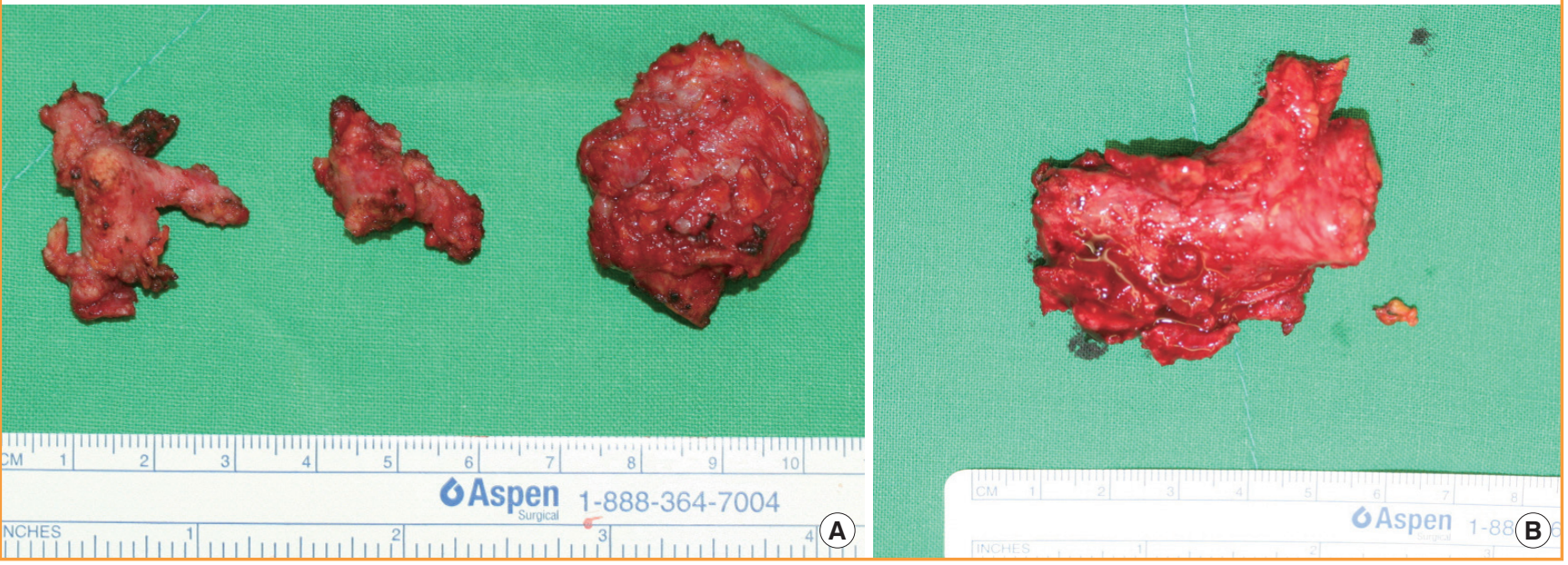




\section{Fig. 3. Histology}

Histopathologic findings show lymphoid hyperplasia with massive eosinophil infiltration $\left(H \& E_{1} \times 200\right)(A)$, exhibiting dense lymphoeosinophilic infiltration in a fibrotic background $\left(H \& E_{1} \times 200\right)(B)$.

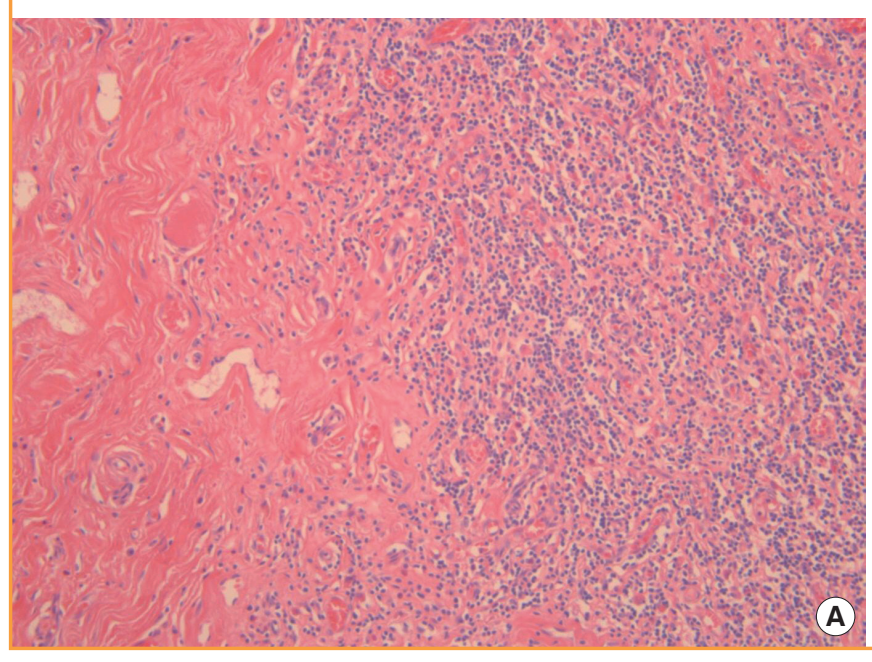

\section{Fig. 4. Facial contours}

Preoperative photograph (A) and postoperative photograph (B). The facial contours were restored to their original form.
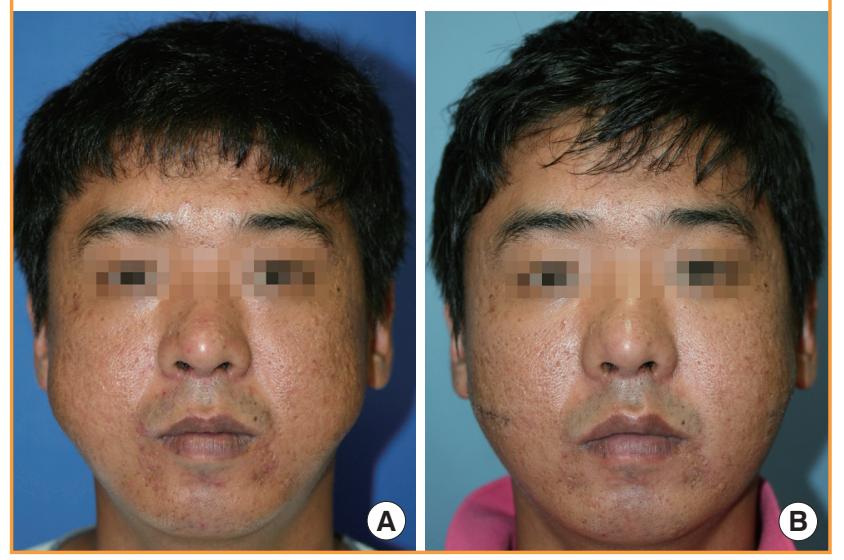

with a decrease in the percentage of peripheral eosinophils to within the normal range ( $4.9 \%$ on postoperative day 5 ). There was no sign of facial nerve injury or relapse for at least 4 years after surgery. The patient's facial contours were restored to normal (Fig. 4), and the patient experienced no pain after recovery.

\section{DISCUSSION}

$\mathrm{KD}$ is a rare benign chronic inflammatory disorder whose cause is unknown [4]. Potential causes include dysfunctional immune regulation, allergic reactions, viral and nonviral infections, and cancer $[3,5]$. The features of $\mathrm{KD}$ include painless, persistent, and self-limiting increases in blood eosinophil and IgE levels. $\mathrm{KD}$ most commonly presents as a unilateral subcutaneous mass

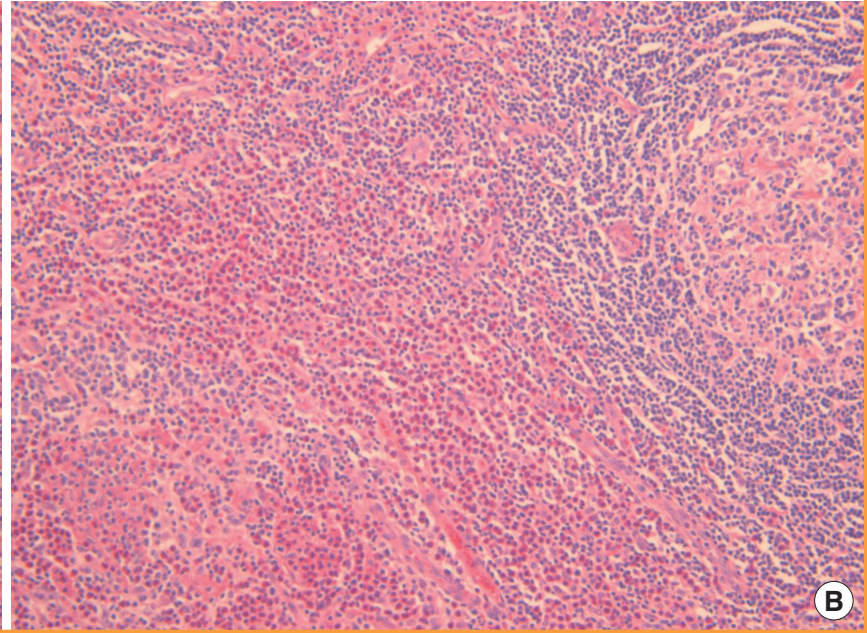

in the head and neck area, and usually affects men in their second and third decades of life $[1,6]$. The male-to-female ratio ranges from 3.5:1.0 to 9.0:1.0 [7]. KD is a benign disorder with no potential for malignant transformation $[4,8]$.

$\mathrm{KD}$ is relatively difficult to diagnose because it rarely occurs and hence is unfamiliar to most physicians and surgeons. The differential diagnoses include malignancies in the head and neck area such as lymphomas, Kaposi sarcomas, low grade angiosarcomas, and nodal metastases, and benign conditions such as tuberculosis and angiolymphoid hyperplasia with eosinophilia (ALHE) $[1,9]$. In the past, ALHE was easily confused with KD and considered a variant of $\mathrm{KD}$. However, these diseases differ histologically: a proliferation of vessels (also known as epithelioid hemangioma) and endothelial lining is present in ALHE, whereas $\mathrm{KD}$ is characterized by localized swelling due to chronic inflammation $[9,10]$. Systemic eosinophilia and lymphadenopathy are rare in cases of ALHE. Further, ALHE usually manifests as multiple papules or nodules that bleed easily, but $\mathrm{KD}$ mostly involves solitary lesions larger than $2 \mathrm{~cm}$ in diameter without any skin problems [2,9]. FNAC can be helpful in some cases, but excisional biopsy is preferred for a definitive diagnosis $[2,3,10]$. As suggested by Park et al. [11], CT and magnetic resonance imaging can be useful for diagnosing $\mathrm{KD}$ in the head and neck area; characteristic imaging results include multiple illdefined enhanced masses within and around the parotid gland with associated regional lymphadenopathy. However, the diagnosis of $\mathrm{KD}$ is difficult to confirm without a pathologic report.

When encountered, it is possible for bilateral symmetric facial masses to be considered as part of the natural shape of a patient's face because they are not common. However, surgeons should 
be aware that some diseases can lead to bilateral parotid lesions. Warthin tumor, acinic cell carcinoma, pleomorphic adenoma, and metastatic tumors are some such examples [2,12-14]. Warthin tumor is the second most common benign neoplasm in the parotid gland, and $6 \%$ of cases of Warthin tumor are bilateral [14]. It is also the most common bilateral parotid tumor, accounting for $85 \%$ of such tumors [13]. It is extremely rare for a disease such as $\mathrm{KD}$ to affect both sides of the face simultaneously.

$\mathrm{KD}$ commonly occurs in combination with renal disease, most notably nephrotic syndrome [2]. The coexisting renal disease may be due to immunocomplex-mediated damage or a Th2dominant immune response disorder [3]. Our patient had no renal involvement despite his long history of disease and did not complain of any symptoms after resection of the masses.

Treatments for $\mathrm{KD}$ include radiation, steroid administration, surgical excision, chemotherapy, and cryotherapy; however, no single treatment of choice has been established $[1,8]$. Each treatment method has advantages and disadvantages. Simple surgical excision, for example, requires less time than other treatments and allows immediate histologic confirmation; however, the disease may recur after surgery $[7,15]$, as well as after other treatments. Steroid therapy and radiotherapy can reduce the size of the mass, but usually do not remove it completely [8], and relapse is not uncommon after steroid therapy discontinuation.

In our case, the patient wanted treatment to be carried out rapidly due to the pain he was experiencing; therefore, total surgical excision was the best option for him. However, if the goal is to avoid scarring, steroid therapy or radiotherapy is the most appropriate first-line choice. As noted above, the limitations of nonsurgical treatment include recurrence after treatment termination. Hence, surgery should be considered after steroid therapy or radiotherapy has sufficiently reduced the size of the mass.

Due to the rarity of $\mathrm{KD}$, only a few studies have addressed its cause, pathogenesis, treatment, and/or prognosis, and more studies are needed. Because KD occurs mainly in Korea, Japan, China, and other regions of East Asia, it seems likely that specific genes are involved. To compensate for the small number of cases at each institution, cooperation among institutions is needed to fully elucidate the causes of and treatments for $\mathrm{KD}$.

In conclusion, $\mathrm{KD}$ is a rare idiopathic chronic inflammatory disorder that rarely presents with bilateral involvement. There are many ways to treat $\mathrm{KD}$, including conservative management, steroid administration, radiotherapy, and surgery. In this symptomatic case, surgical excision was the treatment of choice. However, long-term follow up will be needed because patients with $\mathrm{KD}$ have a constant risk of relapse.

\section{PATIENT CONSENT}

The patient provided written informed consent for the publication and the use of their images.

\section{REFERENCES}

1. Faras F, Abo-Alhassan F, Al-Sebeih K, et al. Kimura disease manifesting as synchronous bilateral parotid swelling in a young middle-eastern patient. Case Rep Surg 2014;2014: 648607.

2. Tseng CF, Lin HC, Huang SC, et al. Kimura's disease presenting as bilateral parotid masses. Eur Arch Otorhinolaryngol 2005;262:8-10.

3. Fouda MA, Gheith O, Refaie A, et al. Kimura disease: a case report and review of the literature with a new management protocol. Int J Nephrol 2011;2010:673908.

4. Shankar T, Myreddy N, Varalaxmi KP. Kimura’s disease: a case report in a child. Indian J Otolaryngol Head Neck Surg 2014;66(Suppl 1):237-41.

5. Mitsui M, Ogino S, Ochi K, et al. Three cases of eosinophilic lymphfolliculoid granuloma of the soft tissue originating from the parotid gland. Acta Otolaryngol Suppl 1996;522: 130-2.

6. Hobeika CM, Mohammed TL, Johnson GL, et al. Kimura's disease: case report and review of the literature. J Thorac Imaging 2005;20:298-300.

7. Gong Y, Zhao Y. A mass under parotid gland: a patient with Kimura disease. J Craniofac Surg 2015;26:595.

8. Yazici D, Tuncer U, Ergin M. Kimura disease of the parotid and retroauricular region: a case report. Arch Otolaryngol Head Neck Surg 2007;133:86-9.

9. Panduranga Kamath M, Bhojwani KM, Bhandarkar AM, et al. Angiolymphoid hyperplasia with eosinophilia of root of nose: a rare phenomenon. J Clin Diagn Res 2014;8:144-5.

10. Nag VK, Nandan D, Bhardwaj M. Kimura’s disease presenting with inguinal lymphadenopathy in an 11-year old girl: a case report. Trop Doct 2015;45:54-6.

11. Park SW, Kim HJ, Sung KJ, et al. Kimura disease: CT and MR imaging findings. AJNR Am J Neuroradiol 2012;33: 784-8.

12. Deveer M, Sahan M, Sivrioglu AK, et al. Bilateral multifocal Warthin tumours. BMJ Case Rep 2013;2013:pii:bcr2013009336.

13. Jia YL, Bishwo SP, Nie X, et al. Synchronous bilateral multifocal acinic cell carcinoma of parotid gland: case report and review of the literature. J Oral Maxillofac Surg 2012;70:e57480.

14. Maiorano E, Lo Muzio L, Favia G, et al. Warthin's tumour: a 
study of 78 cases with emphasis on bilaterality, multifocality and association with other malignancies. Oral Oncol 2002; 38:35-40.

15. Park JS, Jin W, Ryu KN, et al. Bilateral asymmetric superfi- cial soft tissue masses with extensive involvement of both upper extremities: demonstration of Kimura's disease by US and MRI (2008: 12b). Eur Radiol 2009;19:781-6. 